\title{
A Compact UWB-BPF based on Microstrip-to- CPW transition with multiple Transmission zeros
}

\begin{abstract}
Abu Nasar Ghazali' ${ }^{1}$ Mohd Sazid ${ }^{2}$ and B. S. Virdee ${ }^{3}$
${ }^{1}$ (corresponding author) Abu Nasar Ghazali is with the School of Electronics, KIIT University, Bhubaneswar - 751024, Odisha, India. e-mail: anghazali@gmail.com, Contact No. +91-8084398383.

${ }^{2}$ Mohd Sazid is with the Dept. of Electronics and Communication Engineering, Noida Institute of Engineering and Technology, Greater Noida - 201306, U.P., India, e-mail: sazid.anwak@gmail.com

${ }^{3}$ B. S. Virdee is with the Faculty of Life Sciences and Computing, Centre for Communications Technology, London Metropolitan University, London N7, 8 DB, U.K

e-mail: $\underline{\text { b.virdee@,londonmet.ac.uk }}$
\end{abstract}

\begin{abstract}
Proposed in this manuscript is a miniaturized Ultra-Wideband (UWB) bandpass filter (BPF) based on broadside coupled technology wherein microstrip and coplanar waveguide (CPW) are present on either side of the substrate. The ground plane of the proposed BPF contains a short-circuited multiple mode resonator (MMR) based $\mathrm{CPW}$ which is electromagnetically coupled through the dielectric to two open circuited microstrip lines on the top. The MMR is initially designed to allocate its first three resonant modes quasi-equally within the specified UWB spectrum. Later, the back-to-back arrangement of microstrip lines is optimized to generate a tightly coupled broadband response. This configuration generates an UWB response possessing insertion loss $<0.26 \mathrm{~dB}$ in simulation, two transmission zeros at the lower and upper passband edges that improve selectivity and a wide stopband with appreciable attenuation. The predicted theory is validated by testing the simulation result against the measured data of the fabricated prototype. The prototype measures only $14.6 \times 9.2 \mathrm{~mm}^{2}$.
\end{abstract}

Keywords - Bandpass Filter (BPF), broadside coupled, coplanar waveguide (CPW), microstrip-to-CPW transition, ultra-wideband (UWB). 


\section{INTRODUCTION}

Research on design and development of passive Ultra-Wideband (UWB) components have taken an exponential leap since the allocation of 3.1-10.6 GHz spectrum by Federal Communications Commission (FCC) for indoor communications [1]. One such essential component of the UWB system is the UWB bandpass filter (BPF) which unlike the conventional narrow band filters requires understanding of an advanced theory. These theories were put to use in the design of several UWB filters using different principle [2-12]. An initial filter designed in this respect consisted of a microstrip line mounted on a composite lossy substrate to realize attenuation at high frequencies [2], whereas the basic idea of cascading high pass filter with low pass filter to develop an UWB-BPF was used in [3]. Perhaps, the most convenient method used in designing UWB filters was using a multiple mode resonator (MMR) which provides the flexibility of adjusting the bandwidth using its inherent resonant modes [4, 5]. The impedance ratio of lowimpedance central arm and high impedance arms on ends is properly adjusted to simultaneously excite its resonant modes and place them quasi-equally within the passband. Another excellent method of UWB filter construction is using the broadside coupled technology of microstrip-to-coplanar waveguide (CPW) transition [6-12]. The ground plane consists of CPW based open-circuited [6-8] or short-circuited MMR [912] which is excited to place the resonant modes within the UWB passband and then the optimized coupling of the transition generates a smooth passband with possible transmission zeros (TZ). However, most of the structures mentioned above possess several drawbacks in form of absence of TZs [6-9, 12], which leads to poor selectivity [6,8], poor return/insertion loss [6, 8], large size [6-12].

In this manuscript we report a miniaturized UWB-BPF which overcomes the above mentioned shortcomings. The proposed structure is based on microstrip-to-CPW transition with microstrip lines aligned back-to-back on the top and electromagnetically coupled to the short circuited MMR based CPW in the ground (Fig. 1). Initially, the short circuited MMR is optimized in geometry to allocate its first three resonant modes near the lower, central and upper end of requisite UWB spectrum. Later, microstrip lines on top are optimized in dimensions so as to ensure a tight coupling of the transition thereby generating the specified UWB with multiple TZs, minimum insertion loss, high return loss, wide stopband and flat group delay. Commercial full-wave electromagnetic (EM) software IE3D was used to design and optimize the structure on the RT/Duriod 6010 substrate with relative permittivitty $\varepsilon_{r}=10.8$ and height $0.635 \mathrm{~mm}$. The proposed UWB filter is then fabricated to justify its predicted performance in S-magnitudes and group delay. The following sections deal with the design and implementation of the proposed UWB-BPF. 


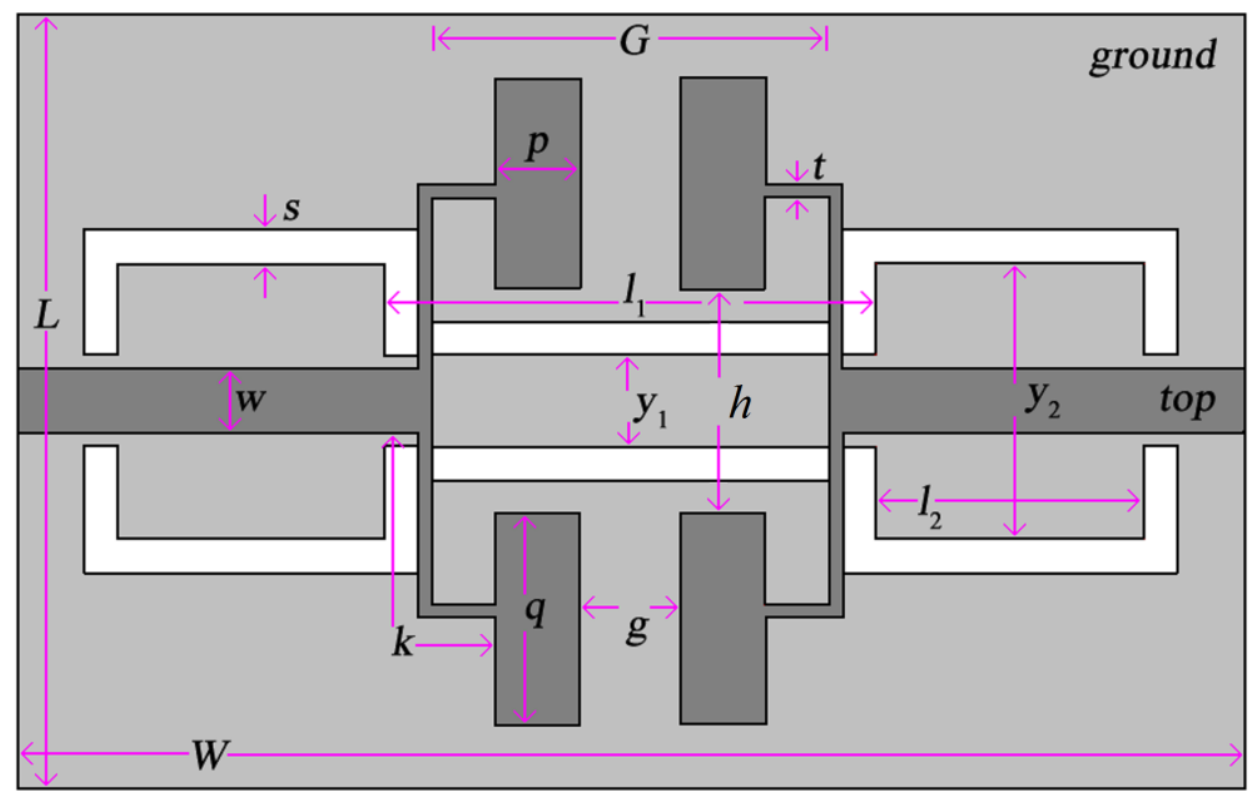

Fig. 1 Microstrip-to-CPW transition based proposed UWB filter. Dark shade conductor and white shade etched part. All dimensions are in $\mathrm{mm}$.

\section{PROPOSED UWB-BPF}

\subsection{CPW BASED SHORT CIRCUITED MMR}

The ground plane of the proposed structure consists of a CPW which houses a short-circuited MMR. The MMR has one high-impedance (narrow) central section and two similar low-impedance (wide) sections at either ends, the geometry and equivalent transmission line model of which are depicted in Fig. 2(a, b). In our analysis we have ignored the two CPW step discontinuities present at the end since their effect is minimum on the UWB characteristics [4]. To utilize the MMR characteristics for design of UWB-BPF, the resonant condition of all the modes must be established. In view of this, the input impedance at the left short-end $\left(Z_{\text {in }}\right)$, looking into the right is derived and depicted in equation (1).

$$
Z_{\text {in }}=j Z_{2} \frac{2\left(K \tan \theta_{1}+\tan \theta_{2}\right)\left(K-\tan \theta_{1} \tan \theta_{2}\right)}{K\left(1-\tan ^{2} \theta_{1}\right)\left(1-\tan ^{2} \theta_{2}\right)-2\left(1+K^{2}\right) \tan \theta_{1} \tan \theta_{2}}
$$

Here, $K=Z_{1} / Z_{2}$ is the ratio of the central and end sections of the MMR. At resonance, $Z_{\text {in }}=0$, and this condition provides some set of equations which can be solved to obtain the lowest resonant frequencies, $f_{1}$, $f_{2}$, and $f_{3}$. For our design, the electrical lengths of three sections are taken as $\theta_{1} \approx \theta_{2} \approx \theta$. Hence, the first three resonant frequencies are obtained from these equations, i.e., 


$$
\theta\left(f_{1}\right)=\tan ^{-1} \sqrt{ } K
$$

or

$$
f_{1}=(c / 2 \pi l) \tan ^{-1} \sqrt{ } K
$$

$$
\theta\left(f_{2}\right)=\pi / 2
$$

or

$$
f_{2}=c / 4 l
$$

$$
\theta\left(f_{3}\right)=\pi-\tan ^{-1} \sqrt{ } K
$$

or

$$
f_{3}=(c / 2 \pi l)\left(\pi-\tan ^{-1} \sqrt{ } K\right)
$$

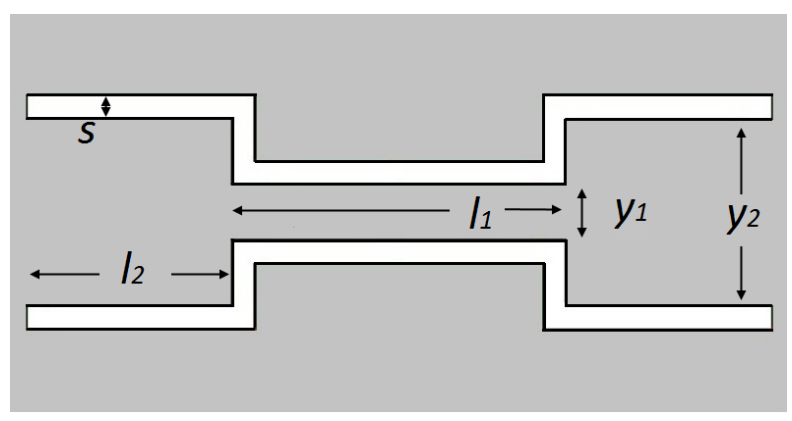

(a)

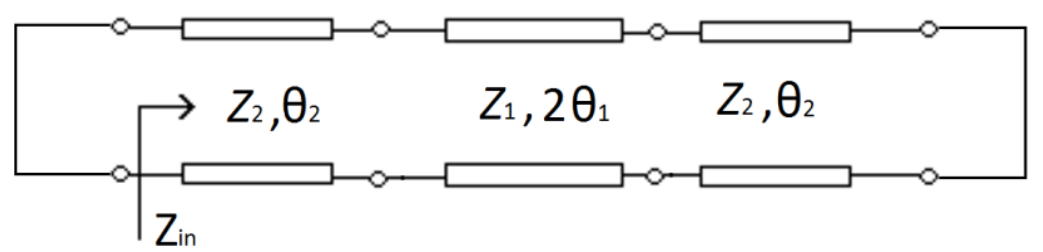

(b)

Fig. 2 (a) Geometry of the MMR based CPW. (b) Equivalent transmission line model.

The above equations depict that the lower and higher frequencies $\left(f_{1}, f_{3}\right)$ are mainly affected by the impedance ration, whereas, the mid frequency $\left(f_{2}\right)$ is a function of lengths of three sections. Fig. 3(a) depicts the relationship of resonant modes relative to the fundamental mode as a function of impedance ratio $K$. From the graph it can be observed that for $K<1$, the resonant modes deviate away from the fundamental mode whereas they draw closer for $K>1$, which essentially is utilized for generating UWB spectrum. In our case, for $l_{1}=5.83 \mathrm{~mm}\left(\approx \lambda_{\mathrm{gCPW}} / 4\right), y_{1}=0.98 \mathrm{~mm}, l_{2}=3.185 \mathrm{~mm}\left(\approx \lambda_{\mathrm{gCPW} 2} / 8\right), y_{2}=3.28 \mathrm{~mm}$ and $s=0.4$ $\mathrm{mm}, K \approx 1.2$. The weak coupling response of the CPW based MMR against variable impedance ratio is plotted in Fig. 3(b). It depicts that with increasing impedance ratio, the position of fundamental resonant mode frequency $\left(f_{1}\right)$ remains somewhat fixed whereas the higher resonant frequencies $\left(f_{2}, f_{3}\right)$ move closer to $f_{1}$. 


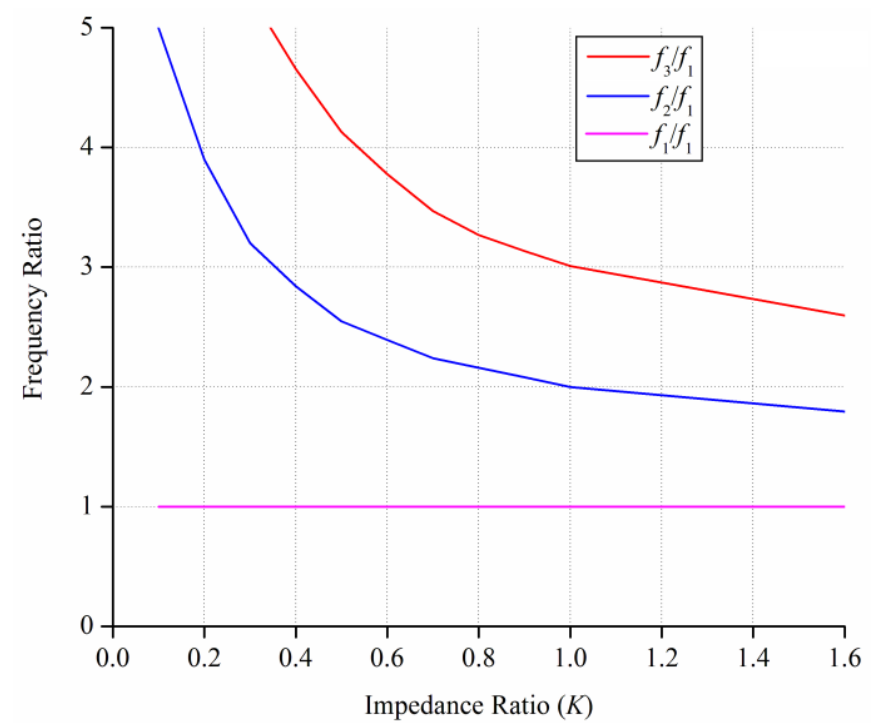

(a)

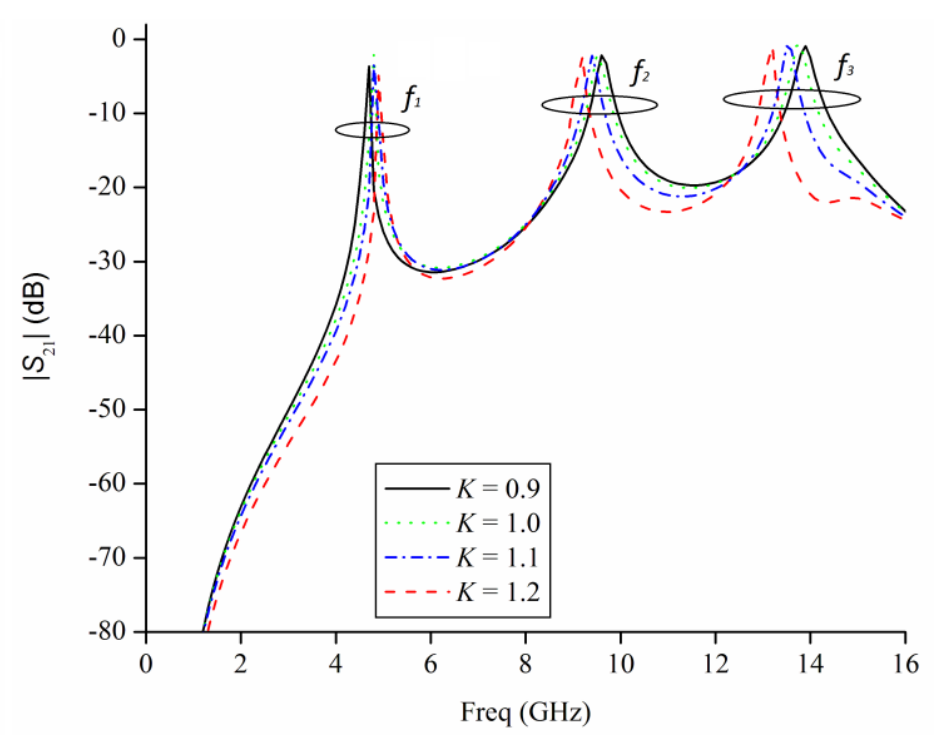

(b)

Fig. 3 (a) Relationship between normalized resonant frequencies and impedance ratio. (b) Weak coupling response for variable impedance ratio.

\subsection{MICROSTRIP-TO-CPW TRANSITION}

Having modeled the CPW, the objective now is to optimize the microstrip-to-CPW transition so as to generate the requisite UWB spectrum with minimum insertion loss and multiple TZs. The transition coupling is capacitive in nature and maximum coupling at the central UWB frequency can be achieved by matching the characteristic impedances of the microstrip line with that of CPW i.e., $Z_{0 \text { (microstrip) }}=2 Z_{0(\mathrm{CPW} 1)}$ [9]. In the proposed structure, for $l_{1}=5.83 \mathrm{~mm}, y_{1}=0.98 \mathrm{~mm}$ and $s=0.4 \mathrm{~mm}, Z_{0(\mathrm{CPW} 1)}=51.58 \Omega$, and for $t$ $=0.15 \mathrm{~mm}, Z_{0 \text { (microstrip })}=81 \Omega$. Ideally $Z_{0 \text { (microstrip) }}=102 \Omega$ would have best suited the above relation, however, $81 \Omega$ is used because it provides wider bandwidth, proper upper TZ and plunged stopband with negligible effect to other frequency characteristics as seen in Fig. 4. Also, for $Z_{0 \text { (microstrip) }}=102 \Omega$, the thickness of microstrip lines, $t=0.12 \mathrm{~mm}$ is little difficult to fabricate. From the simulated response of Fig. 4 , it can be observed that the passband extends from 3.05-10.7 GHz with return/insertion loss better than 15/0.54 dB. The two TZs at $0.95 \mathrm{GHz}$ and $11.7 \mathrm{GHz}$ provide a sharp roll-off $>34$ and $48 \mathrm{~dB} / \mathrm{GHz}$ respectively at lower and upper passband edges. The third TZ at $16 \mathrm{GHz}$ ensures a wide and deep stopband with attenuation greater than $25 \mathrm{~dB}$.

Figs. 6a and $6 \mathrm{~b}$ depict the variation of frequency characteristics of the UWB-BPF for vertical $(h)$ and horizontal $(\mathrm{g})$ separation between the microstrip lines respectively. The variation in vertical separation $(h)$ causes the TZs at the higher passband end and in the stopband to vary without affecting the position of lower TZ. Also, the horizontal separation (g) controls the position of all three TZs. The optimum values of 
$h$ and $g$ bring about proper positioning of all three TZs which lead to better passband and stopband characteristics. The attenuation level of stopband can be adjusted by tuning the values of central and end sections of the CPW, $y_{1}$ and $y_{2}$, respectively, as depicted in Fig. 6a and 6b. The optimized dimension of the UWB-BPF is provided in Table I.

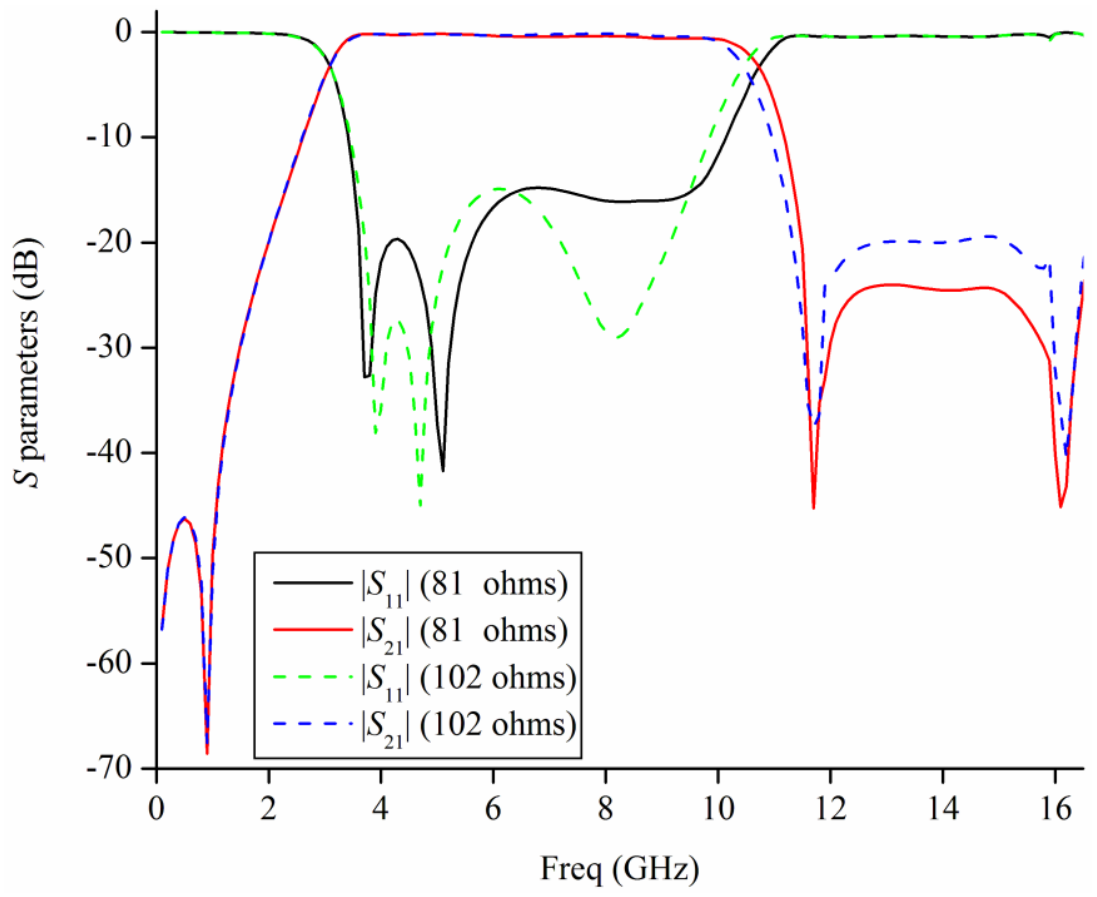

Fig. 4 Comparative frequency characteristics for variable impedance of CPW.

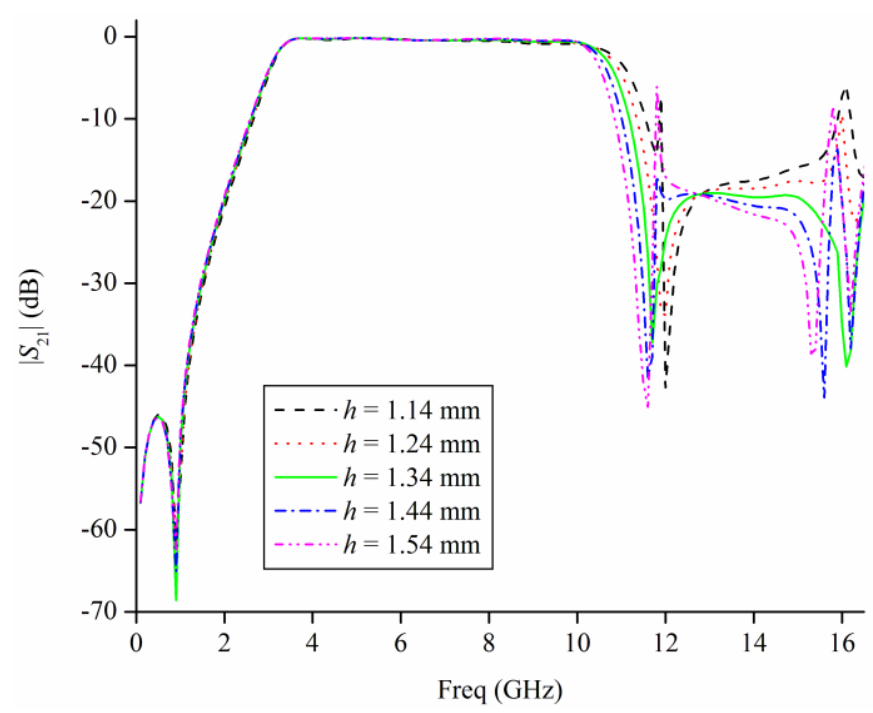

(a)

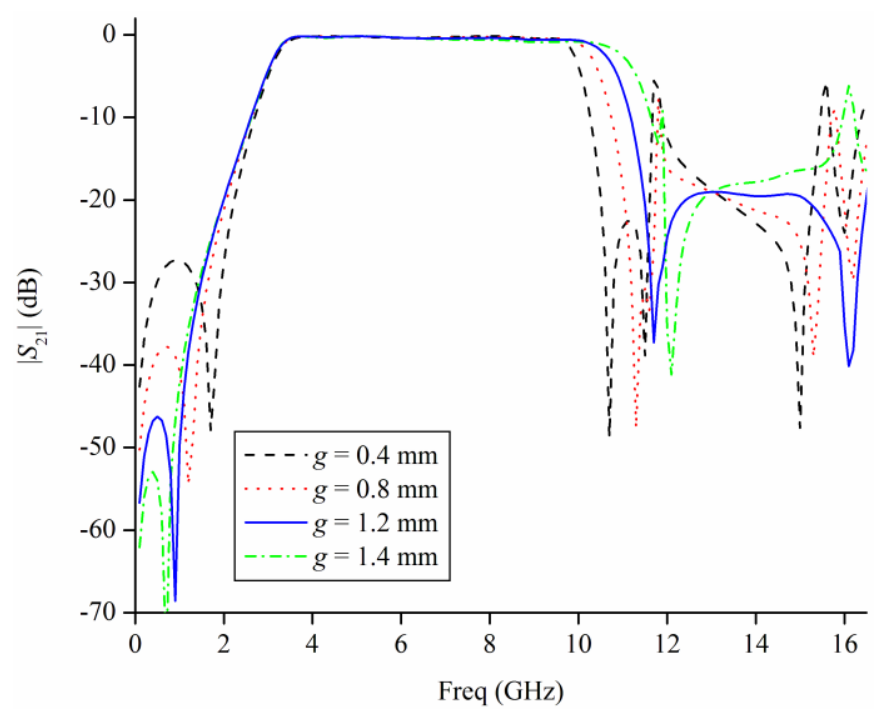

(b)

Fig. 5 Variable transmission characteristics for (a) $h$ (b) $g$. 


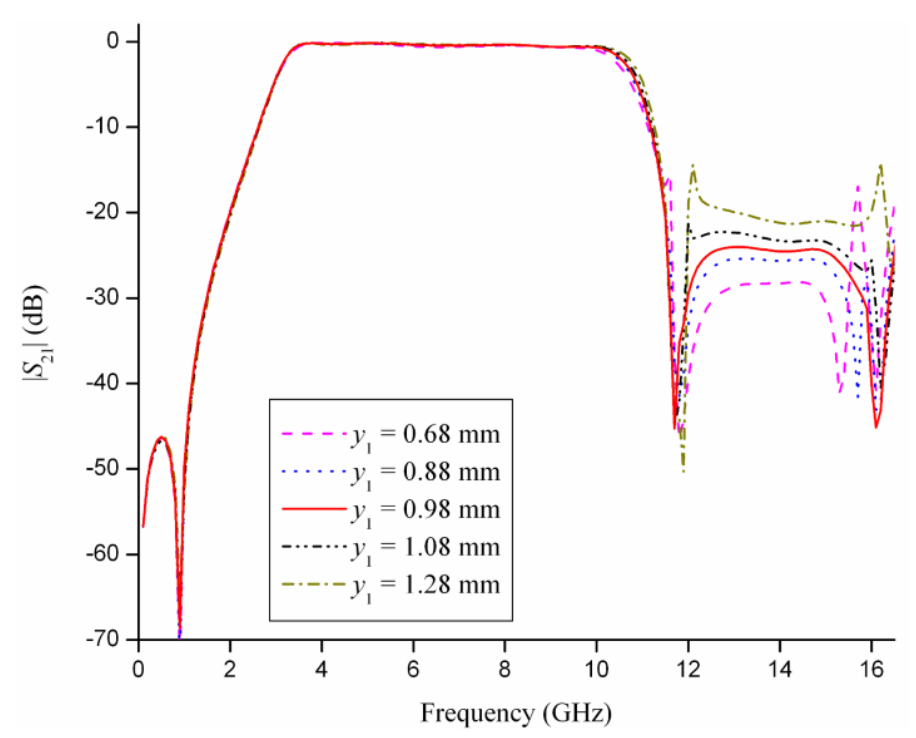

(a)

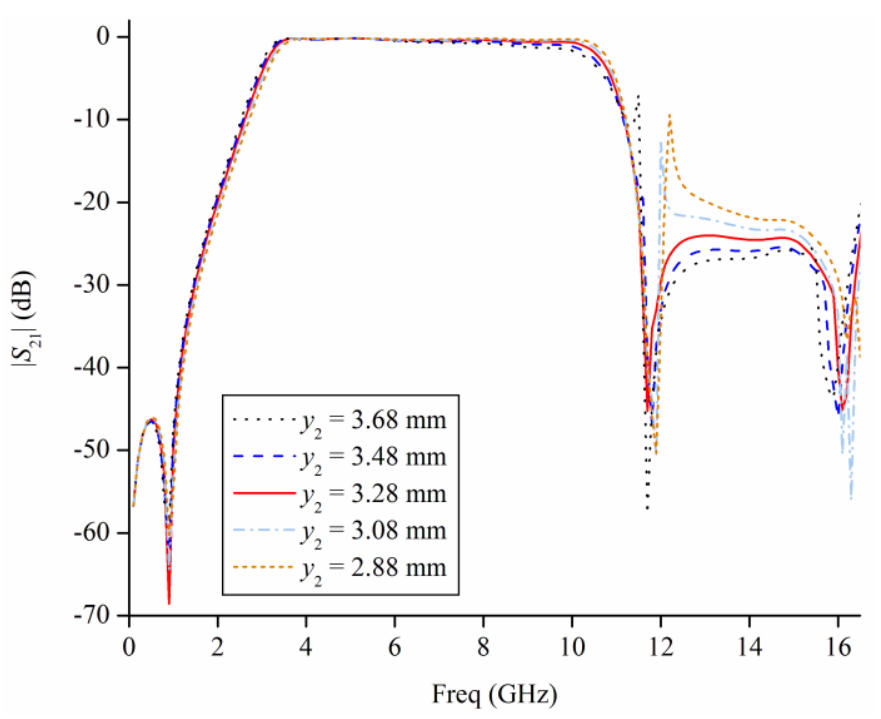

(b)

Fig. 6 Variable frequency characteristics for (a) variable $y_{1}(\mathrm{~b})$ variable $y_{2}$.

Table I

Optimized dimension of the proposed UWB-BPF.

\begin{tabular}{|c|c|c|c|c|c|c|c|c|}
\hline Parameters & $L$ & $W$ & $G$ & $l_{1}$ & $l_{2}$ & $y_{1}$ & $y_{2}$ & $h$ \\
\hline $\begin{array}{c}\text { Dimension } \\
(\mathrm{mm})\end{array}$ & 14.6 & 9.2 & 4.75 & 5.83 & 3.185 & 0.98 & 3.28 & 1.34 \\
\hline Parameters & $q$ & $g$ & $s$ & $p$ & $t$ & $w$ & $k$ & -- \\
\hline $\begin{array}{c}\text { Dimension } \\
(\mathrm{mm})\end{array}$ & 2.5 & 1.2 & 0.4 & 1 & 0.15 & 0.76 & 3.115 & -- \\
\hline
\end{tabular}

\section{EXPERIMENTAL VERIFICATION}

To verify the predicted performance, the structure is fabricated and its response measured using Agilent Vector Network Analyzer N5230A. Fig. 7a shows that the passband measured extends from 3 to $10.9 \mathrm{GHz}$ with insertion loss less than $1.4 \mathrm{~dB}$ and return loss greater than $15 \mathrm{~dB}$. Stopband observed is deep with attenuation greater than $19 \mathrm{~dB}$ and wide till $16 \mathrm{GHz}$. From Fig. 7b, the measured group delay is observed to vary between 0.21 to $0.36 \mathrm{~ns}$ in the passband indicating good linearity. The variations in simulated and measured data is possibly due to loss in two SMA connectors and finite substrate size. The data measured is in reasonable agreement with the simulated response and also accords with the FCC defined UWB mask for indoor wireless communication. The designed filter is less than one guided wavelength at central UWB frequency $(6.85 \mathrm{GHz})$ which depicts is compact nature. The proposed structure is compared with other 
recent broadside coupled UWB filters in Table II, from which it can be seen that the proposed structure is compact compared to rest [6-12]. Also, it meets the requisite FCC specified UWB spectrum limitation with appreciable frequency characteristics.

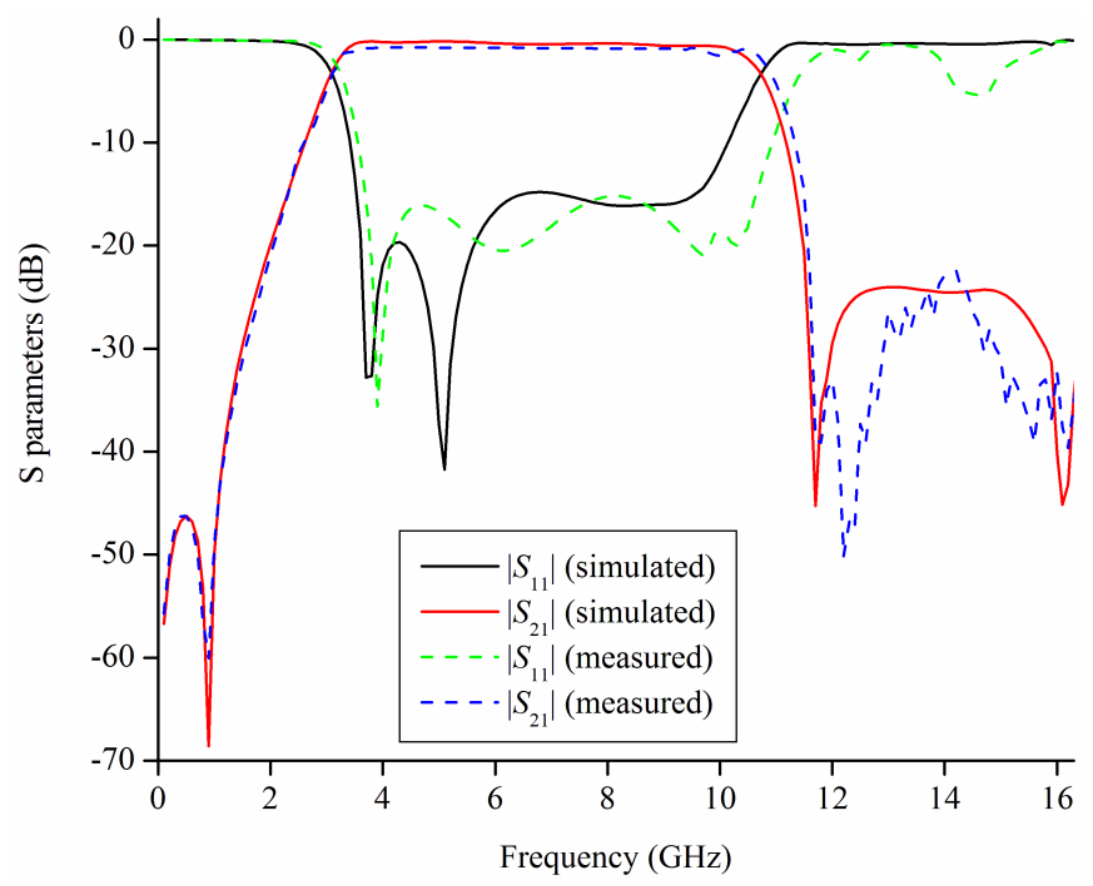

(a)

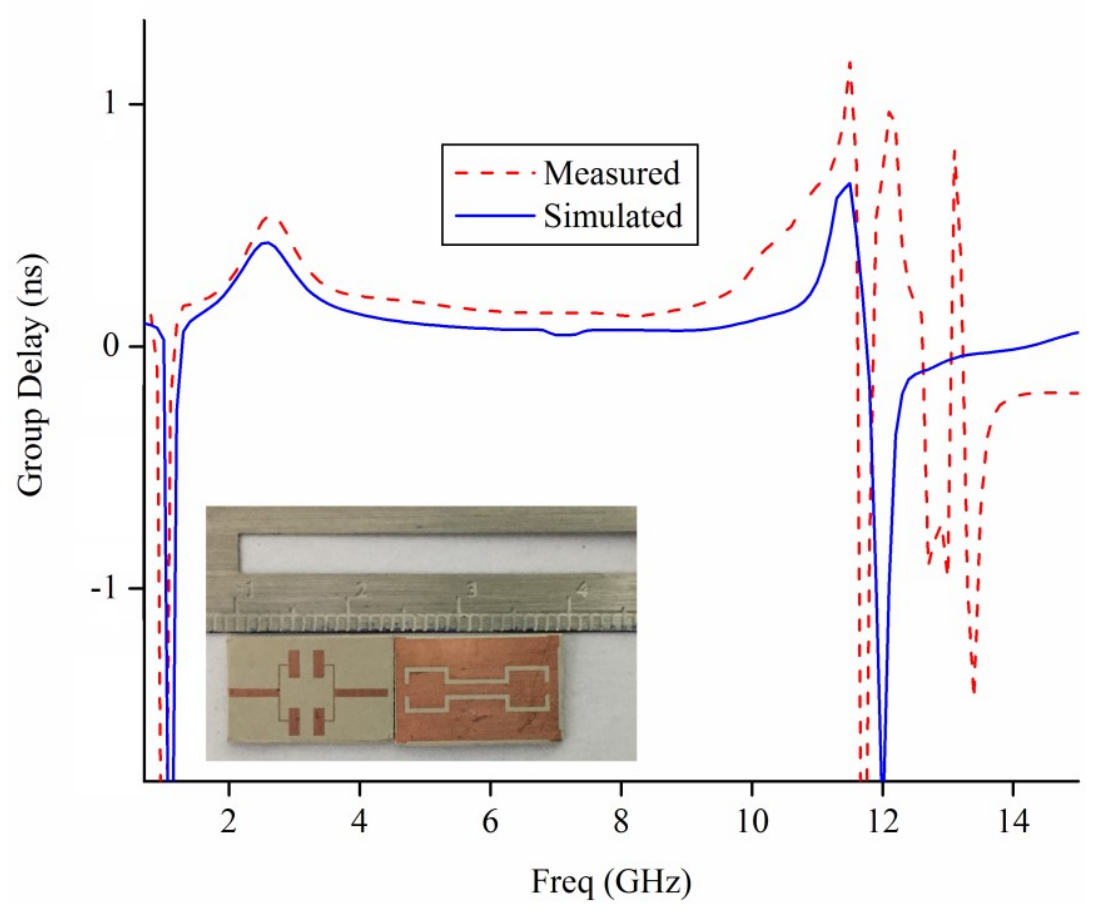

(b)

Fig. 7 Comparative measured and simulated frequency characteristics. (a) $S$ parameters. (b) Group delay. 
Table II

Comparison of this work with other known structures

\begin{tabular}{|c|c|c|c|c|}
\hline Ref. & $\begin{array}{c}\text { Passband } \\
(\mathrm{GHz})\end{array}$ & $\begin{array}{c}\text { Stopband }(\mathrm{GHz}) / \\
\text { Attenuation }(\mathrm{dB})\end{array}$ & $\begin{array}{c}\mathrm{IL}(\mathrm{dB}) / \\
\mathrm{RL}(\mathrm{dB})\end{array}$ & $\begin{array}{c}\text { Size } \\
(\mathrm{mm} \times \mathrm{mm})\end{array}$ \\
\hline$[6]$ & NA & $13 / 18$ & $<1 />9$ & $>26.9 \times 16$ \\
\hline$[7]$ & $3.1-10.6$ & $20 / 15$ & $<0.5 />15$ & $12 \times 18$ \\
\hline$[8]$ & NA & $12 / 20$ & $<0.5 />13$ & $23.98 \times 4.96$ \\
\hline$[9]$ & $3.1-10.6$ & $15.5 / 15$ & $<1.8 />10$ & $>34 \times 12$ \\
\hline$[10]$ & $2.9-10.95$ & $12.5 / 19$ & $<0.68 />14$ & $14.8 \times 7.3$ \\
\hline$[11]$ & $2.7-10.6$ & $12.5 / 19$ & $<0.41 />16$ & $14.8 \times 9.6$ \\
\hline$[12]$ & $3.07-10.51$ & $30 / 19$ & $<1 />17$ & $27 \times 27$ \\
\hline $\begin{array}{c}\text { This } \\
\text { work }\end{array}$ & $3-10.9$ & $16 / 19$ & $<1.4 />15$ & $14.6 \times 9.2$ \\
\hline
\end{tabular}

Ref : References; IL : Insertion loss; RL : Return loss; NA : Not available

\section{CONCLUSION}

The manuscript proposes a novel and compact UWB filter based on the broadside coupled hybrid microstrip/CPW transition. The short circuited MMR in ground is excited to equally space its resonant modes within the UWB passband and the broadside coupling of microstrip/CPW transition is optimized to generate a good UWB response. The presence of dual TZs at the passband edges improve the filter selectivity whereas a third TZ widens and suppresses the stopband. As such, an UWB-BPF with good frequency characteristics having improved insertion loss/ return loss $(<0.26 \mathrm{~dB} />15 \mathrm{~dB})$ and flat group delay is achieved. Also, the proposed structure has an overall length of $14.6 \mathrm{~mm}$, which is less than the guided wavelength at central UWB frequency.

\section{ACKNOWLEDGEMENTS}

The authors appreciate Sunrays Circuits, Bangalore, for their generous help in filter's fabrication. 


\section{REFERENCES}

[1] Revision of Part 15 of the Commission's Rules Regarding Ultra-Wideband Transmission Systems', First Note and Order Federal Communications Commission, ET-Docket 98-153, 2002.

[2] Saito A, Harada H and Nishikata A. Development of bandpass filter for ultra wideband (UWB) communication. Proc. IEEE Conf. Ultra Wideband Syst. Tech. 2003: 76-80.

[3] Tang CW and Chen MG. A microstrip ultra-wideband bandpass filter with cascaded broadband bandpass and bandstop filters. IEEE Trans. Microw. Theory Tech. 2007; 55: 2412-2418.

[4] Gao J, Zhu L, Menzel W and Bogelsack F. Short-circuited CPW multiple-mode resonator for ultrawideband (UWB) bandpass filter. IEEE Microw. Wireless Compon. Lett. 2006; 16: 104-106.

[5] Zhang T, Xiao F, Bao J and Tang X. Compact ultra-wideband bandpass filter with good selectivity. Electron. Lett. 2016; 52: 210-212.

[6] Wang $\mathrm{H}$, Zhu L, and Menzel W. Ultra-Wideband bandpass filter with hybrid microstrip/CPW structure. IEEE Microw. Wireless Compon. Lett. 2005; 15: 844-846.

[7] Abbosh A, Bialkowski M and Ibrahim S. Ultra-wideband bandpass filters using broadside-coupled microstrip-coplanar waveguide. IET Microw. Antennas Propag. 2011; 5: 764-770.

[8] Jadhav JB and Deore PJ. A compact planar ultra-wideband bandpass filter with multiple resonant and defected ground structure. Int. J. Electron. Commun. (AEÜ). 2017; 81: 31-36.

[9] Baik JW, Lee TH, and Kim YS, UWB Bandpass Filter Using Microstrip-to-CPW Transition with Broadband Balun. IEEE Microw Wireless Compon Lett. 2007; 17: 846-848.

[10] Ghazali AN, Sazid M and Pal S. A Compact Broadband Balun based UWB-BPF with Minimum Insertion Loss and Sharp Selectivity. Electron. Lett. 2015; 51: 1174 -1175.

[11] Ghazali AN, Sazid M and Pal S. A Miniaturized Microstrip-to-CPW Transition based UWB-BPF with Sharp Roll-Off and Minimum Insertion Loss. Microw. Opt. Tech. Lett. 2016; 58: 289-293.

[12] Sakotic Z, Bengin VC, Jankovic N, Compact circular-patch-based bandpass filter for ultra-wideband wireless communication systems. Int. J. Electron. Commun. (AEÜ). 2017; 82: 272-278. 